\title{
Políticas Públicas. Una introducción a la teoría y la práctica del análisis de las políticas públicas, por Wayne Parsons, (2007) Argentina: Edgard Elgar y FLACSO-México.
}

$\mathrm{M}$

uchas cosas distinguen y hacen único el libro titulado Políticas Públicas. Una introducción a la teoría y práctica del análisis de políticas públicas. Su autor, Wayne Parsons se dio a la tarea de reunir en una sola publicación los aspectos más relevantes del conocimiento que se ha producido alrededor de las políticas públicas. En su libro da cuenta de los temas y debates teóricos centrales de esta nueva disciplina, además de ofrecer múltiples referentes bibliográficos y detalles sobre estudios de caso que ilustran la riqueza del enfoque de políticas públicas, pues muestran no sólo cómo se aplica sino, sobre todo, qué explicaciones y respuestas puede ofrecer.

La visión que se plantea en el texto es integral, marcado por el balance entre las disciplinas que moldean el enfoque de las políticas públicas, destacadamente la ciencia política y la economía. En este sentido, no hay en el texto una perspectiva, teoría o modelo que domine la visión del análisis de políticas públicas, sino la reivindicación de la necesaria contextualización de diferentes marcos analíticos y métodos desde los cuales el análisis de tales políticas intenta capturar la complejidad económica, política, social y cultural que incide en el desarrollo de las políticas públicas.

A lo anterior se suma uno de los rasgos más valiosos del libro: poner en claro dos ángulos del enfoque de las políticas, con frecuencia ignorados en la bibliografía sobre la materia, a saber: el análisis del proceso de las políticas, por una parte y, por la otra, el análisis en y para el proceso de políticas. Aunque ambos están intrínsecamente ligados, su especificidad determina su naturaleza y fin. Mientras el análisis del proceso de las políticas tiene fines heurísticos y se concentra, sobre todo, en explicar cómo se definen los problemas, cómo se establecen las agendas, cómo se formulan las políticas, cómo se toman las decisiones y cómo se implementan y evalúan las políticas; el segundo, el análisis en y para el proceso de las políticas, tiene fines prescriptivos, esto es, pretende, sobre todo, apoyar la toma de decisiones, e incluye el uso de herramientas analíticas, de investigación y argumentación en la definición de los problemas, la selección de alternativas, la implementación y la evaluación.

A través de una exposición excepcionalmente clara y con un lenguaje accesible, Parsons permite al lector entrar en contacto con los principales marcos analíticos y teóricos empleados para estudiar los problemas públicos y los procesos alrededor de las políticas públicas. Esto posibilita mostrar algunos de los rasgos distintivos de la 'ciencia de las políticas', a saber: su carácter multidisciplinario, multimetódico, su orientación hacia la solución de problemas (o problem solving) y su preocupación por mapear el contexto del proceso de las políticas, las opciones, los resultados y el impacto. 
La edición en español de Public Policy An introduction to the theory and practice of policy analysis contiene 5 capítulos. El primero, funciona como una auténtica introducción al enfoque de políticas públicas, parte desde la definición de los conceptos de lo público y las políticas, y estudia la forma como ha evolucionado este enfoque; los tipos de análisis y analistas que existen; y los principales modelos que se han desarrollado. A partir del segundo capítulo, analiza propiamente los principales temas y debates que han definido el campo de las políticas públicas, y se adhiere a los que reivindican la utilidad del modelo del ciclo de las políticas públicas, aunque no deja de reconocer algunas de sus limitaciones. Como herramienta heurística, el ciclo - $\mathrm{O}$ modelo por etapas - brinda una estructura racional que permite aprehender la complejidad de la realidad hasta ponerla en una forma manejable. Cada una de sus 7 etapas -surgimiento del problema, definición del problema, identificación de soluciones o respuestas alternativas, evaluación de opciones, selección de una opción de política, implementación y evaluación- provee un contexto en el que podemos utilizar uno o varios marcos analíticos para encontrar explicaciones plausibles de los problemas considerados como públicos, lo que enrique el análisis.

Parsons describe ampliamente cada etapa del ciclo, así como los marcos o enfoques relevantes para cada una. Así, por ejemplo, en el caso de la definición de los problemas, además de mencionar en qué consiste esta etapa, expone los enfoques positivista, sociológico o constructivista y el de la opinión pública. Pese a sus diferencias respecto a la forma de "leer" la realidad, hoy más que considerarlos excluyentes, parece pertinente incluir sus aportaciones para enriquecer la ardua tarea de dilucidar qué cuenta como un problema público y cómo un problema es definido. Para la fijación de la agenda - un tema central en el enfoque de políticas públicas y casi olvidado por la administración públicaParsons expone dos enfoques que nos ofrecen visiones inicialmente en disputa pero en realidad claramente complementarias. Por un lado, el enfoque pluralista de Dahl y Lindblom (Dahl y Lindblom, 1953; Dahl, 1961), que reivindica la competencia entre grupos provenientes de distintas posiciones sociales; $y$, por otro, el enfoque del manejo de la política, o mobilization of vias de Schattschneider, Cobb y Elder, quienes sostienen que tanto la definición de los problemas, como la fijación de la agenda son resultado de las reglas, estructuras y límites puestos por los actores públicos y privados más poderosos (Schattschneider, 1960; Cobb and Elder, 1972).

El texto tiene todo un capítulo - el tercero- dedicado al análisis de las decisiones, que es tal vez el asunto más debatido en el área de las políticas públicas. Ciertamente, la toma de decisiones - o hechura de las políticas, como le llama Luis F. Aguilar (Aguilar, 1992) - requiere entender la manera en que los hechos y los valores interactúan, esto es, la manera en que las creencias, ideas e intereses de cada uno de los múltiples actores que intervienen en las decisiones se entrelazan con la información, los hechos y lo que perciben como la "realidad". Esto ayuda a comprender por qué difícilmente hay una única explicación de las decisiones, y de la forma en que se adoptan. En la práctica, como se sabe, suelen contender varias. Ahora bien, entre los enfoques y categorías relevantes por Parsons para analizar la toma de decisiones destacan el del poder, el de la racionalidad, el de la elección pública y sus diferentes líneas internas, el institucional y el psicológico que, como en el caso de otras etapas del ciclo de las políticas, suelen brindarnos perspectivas diferentes pero complementarias que enriquecen el análisis de las acciones pasadas o por venir.

La implementación y la evaluación, que por años fueron ignoradas por los analistas, forman parte del cuarto capítulo, dedicado al análisis de las acciones de respuesta (delivery analysis). Según señala Parsons, fue hasta 1970 que comenzó a parecer evidente que muchos programas y políticas gubernamentales no sólo no se habían desarrollado como se esperaba, sino que no habían obtenido los resultados deseados o incluso habían producido impactos inesperados. Por 
ello, los estudiosos de las políticas públicas cambiaron su atención de los inputs y procesos - en los que se habían especializado por años- hacia los outputs and outcomes, esto es, los resultados y sus impactos. Parsons dedica algunos apartados del cuarto capítulo del libro para describirlas y mostrar los principales enfoques que se utilizan, entre los que destacan los del mercado $y$ gerenciales, que ofrecieron una alternativa radical al enfoque jerárquico que dominó la implementación y evaluación por años.

El último y quinto capítulo es una contribución que hemos realizado a la primera edición en español del texto Public Policy An introduction to the theory and practice of policy analysis. Este capítulo incluye una serie de casos latinoamericanos de política pública, que ejemplifican el éxito y/o fracaso de algunas políticas en la región latinoamericana; los problemas de diseño, implementación y evaluación de otras, algunos esfuerzos de análisis comparado de políticas públicas, etcétera. Asimismo, se han incluido tanto a lo largo del libro como en la bibliografía general los referentes bibliográficos de aquellos textos mencionados por W. Parsons que han sido traducidos al español. Lo anterior con el propósito de hacer más accesible la bibliografía complementaria para el lector.

La riqueza del enfoque de las políticas públicas que se advierte en el libro de Parsons contrasta con la relativamente reciente aparición de la disciplina y su desarrollo aún precario, en contextos poco democráticos o en aquellos en los que la democracia se encuentra aún en vías de consolidación. Como se sabe, el campo de las políticas públicas nació en Norteamérica bajo los supuestos de un entorno democrático estable y de la existencia de numerosas organizaciones independientes del gobierno, capacitadas tanto para la crítica como para la participación en el ejercicio del gobierno y, en general, en la marcha y directrices de las decisiones públicas. Semejante panorama dista de existir en América Latina, por citar un ejemplo. Las democracias emergentes de la tercera ola de democratización o posteriores están aún construyendo las instituciones democráticas que componen los cimientos de una sociedad fuerte y organizada. Es más, aunque celebran elecciones competitivas - lo que constituye el piso básico de cualquier democracia, que puede ser más pero no menos- no en todos los casos han logrado conquistar la estabilidad democrática necesaria para desvanecer los riesgos del regreso al autoritarismo.

Así, el estado actual de la democracia en América Latina ofrece pocos referentes empíricos que permitan realizar una más clara y cotidiana aplicación del enfoque de las políticas a lo que hace o deja de hacer el gobierno. Esto explica en alguna medida el escaso desarrollo de la teoría y práctica de las políticas públicas y el todavía limitado acervo de textos y documentos sobre este campo en español.

La importancia de la edición en español del texto de W. Parsons radica en que pone al alcance del público hispanohablante una revisión exhaustiva del saber en la materia que ha sido producido, principalmente en Estados Unidos y la Gran Bretaña, por más de medio siglo. Esto es doblemente relevante en un contexto en el que las naciones latinoamericanas están culminando su proceso de transición a la democracia y la legitimidad de los gobiernos está cada vez más ligada a su capacidad y a su desempeño en la atención de los problemas públicos. Así, Public Policy An introduction to the Theory and Practice of Policy Analysis, llega al mercado hispanoamericano en un momento en el cual sus aportaciones pueden resultar de gran valor tanto para el desarrollo de la naciente disciplina en la región como para la propia práctica gubernamental.

Irma Méndez de Hoyos Profesor-investigador Facultad Latinoamericana de Ciencias Sociales sede México.

Tomislav Lendo Fuentes Funcionario público, Presidencia de la República. 